\title{
Corrigendum
}

\section{On the complexity of the successivity relation in computable linear orderings}

[J. Math. Log., Vol. 10, Nos. 1 \& 2 (2010), pp. 83-99, DOI: 10.1142/S0219061310000936]

Rodney G. Downey

School of Mathematics, Statistics and Operations Research Victoria University of Wellington

Wellington, New Zealand

Rod.Downey@mcs.vuw.ac.nz

Steffen Lempp

Department of Mathematics, University of Wisconsin

Madison, Wisconsin 53706-1388, USA

lempp@math.wisc.edu

Guohua Wu

School of Physical and Mathematical Sciences

Nanyang Technological University

Singapore 637371, Republic of Singapore

guohua@ntu.edu.sg

Published 17 July 2017

We indicate how to fix an error in the proof of the Main Theorem of our original paper pointed out to us by Zubkov.

The main result of our paper [2] reads as follows:

Main Theorem. Let $\mathcal{A}$ be an infinite computable linear ordering with infinitely many successivities. Suppose that $C$ is any c.e. set with $\operatorname{Succ}(\mathcal{A}) \leq_{T} C$. Then there is a computable linear ordering $\mathcal{B}$ isomorphic to $\mathcal{A}$ whose successivity relation has Turing degree $\operatorname{deg}_{T}(C)$.

Now, Chubb, Frolov and Harizanov [1] had already handled the case of successivities occurring arbitrarily far to the right in a linear order $\mathcal{A}$ without right endpoint; and their proof can easily be adapted to the case of any point in $\mathcal{A}$ (including the "virtual limit points" $+\infty$ and $-\infty$ ) being a limit point of successivities; more precisely, their proof can handle the case when, given a fixed $a \in A \cup\{+\infty\}$, for any $b<_{\mathcal{A}} a$, there are infinitely many successivities in the interval $(b, a)$, and, 
symmetrically, the case when, given a fixed $a \in A \cup\{-\infty\}$, for any $b>_{\mathcal{A}} a$, there are infinitely many successivities in the interval $(a, b)$.

Moreover, Frolov [3] had already handled the case of non- $\eta$-like and of strongly $\eta$ like linear orderings, so our paper concentrated on the case of $\eta$-like linear orderings without infinite strongly $\eta$-like intervals.

Zubkov (personal communication) pointed out errors as well as a missing case in our proof, both of which items we aim to correct here.

Let us first start with the errors in the proof of our Main Theorem in 2] in the case of a linear order $\mathcal{A}$ in which any infinite interval is $\eta$-like but not strongly $\eta$-like. Specifically, in the description of the full $\mathcal{W}_{a}$-strategy in Sec. 3.2.1 there, the first bullet handles the case of a strategy $\sigma$ acting for the first time since its most recent initialization. If there is a strategy $\sigma_{0}$ to the left as described in the construction there, which was the most recent strategy working on this requirement that has not been initialized since, then we guess at the size of the maximal block containing a by measuring $L$ and $R$, respectively, as if $\sigma_{0}$ was measuring. Then, however, we create $L$ many new elements immediately to the left of $a$, and $R$ many new elements immediately to the right of $a$. As pointed out by Zubkov, this can lead to too many new elements being created by strategies to the right of the true path.

Instead, in the first bullet of Sec. 3.2.1, if $\sigma_{0}$ exists and we measure $L$ and $R$ as described there, we should reuse as many old elements as possible that were used by $\sigma_{0}$ at stage $s_{0}$; i.e. if at stage $s_{0}, \sigma_{0}$ measured $L_{0}$ many elements in the maximal block to the left of $a$ and $L>L_{0}$, then we should reuse the $L_{0}$ many elements from $\sigma_{0}$ immediately to the left of $a$ for $\sigma$ as well, and $\sigma$ should only create $L-L_{0}$ many new elements immediately to the left of these; similarly, if $L \leq L_{0}$, then $\sigma$ should not create any new elements but reuse the $L_{0}$ many elements immediately to the left of $a$ used by $\sigma_{0}$. The same also applies to elements immediately to the right of $a$. (A minor typo also occurs right at the beginning of 3.2 .1 as well as in the first bullet of 3.2.1: $\iota_{\sigma}^{-}(a)$ should read as $\iota_{\sigma^{-}}(a)$.)

The same analysis also applies — mutatis mutandis — to the full $\mathcal{S}_{b}$-strategy.

With these two changes, Lemmas 3.2(4)(b) and 3.2(5)(c) are now correct as stated.

Now let us turn to the missing case to establish our Main Theorem in [2], namely, the case when the linear order $\mathcal{A}$ is $\eta$-like but has no infinite strongly $\eta$-like interval nor a limit point of successivities handled à la Chubb et al. It is not hard to check that any such order must contain an infinite interval of the form

$$
\sum_{i \in \omega}\left(\eta+m_{i}\right)+\sum_{i \in \omega^{*}}\left(n_{i}+\eta\right),
$$

where $m_{i}, n_{i} \in \omega$, and $\lim \sup _{i} m_{i}=\infty$ or $\lim \sup _{i} n_{i}=\infty$ (or both). For our proof below, we will actually only need the property that for all $i \in \omega, m_{i}>1$, or for all $i \in \omega, n_{i}>1$; the important property of such a linear order $\mathcal{A}$ for us is that given any $a \in A$, exactly one of the intervals $(-\infty, a)$ or $(a, \infty)$ contains infinitely many 
successivities, and so we can guess at the location of the finitely many successivities in the other interval.

As in the construction of our paper [2], we will build a computable copy $\mathcal{B}$ of $\mathcal{A}$ as well as, given a c.e. $\operatorname{set} C \geq_{T} \operatorname{Succ}(\mathcal{A})$, a computable functional $\Gamma$ with $\Gamma^{\operatorname{Succ}(\mathcal{B})}=C$. When defining $\Gamma^{\operatorname{Succ}(\mathcal{B})}(i)$ for some argument $i$, we search for a new successivity $(a, b)$ in $\mathcal{A}$ and use its image in $\mathcal{B}$ in the oracle of $\Gamma$, together with various other successivities handed down from higher-priority strategies. As mentioned above, this pair $(a, b)$ now splits the current "working interval" $I$, say, into two halves such that exactly one of them contains only finitely many successivities. So for each half, we guess a sequence of numbers $\left(a_{0}, a_{1}, \ldots, a_{n}\right)$ such that the intervals to the left of $a_{0}$, to the right of $a_{n}$, and between $a_{i}$ and $a_{i+1}$ are either empty or densely-ordered. We know that on one side, we will eventually be able to find such a sequence, and identifying the correct sequence is $\Pi_{2}^{0}$.

When we have found this sequence, we first of all know that the other half of $I$ contains infinitely many successivities so that we can use it as the working interval for further definitions of $\Gamma$. Furthermore, such guesses of tuples with dense or empty intervals in-between can naturally be ordered so that during the construction, the leftmost guess visited infinitely often will be the correct one. Finally, after having identified the points of such a sequence on one side correctly, we may infinitely often think that the dense intervals actually contain only finitely many more points, or that the other side has an (incorrect) sequence. But in each case, $\Gamma$-definitions based on these incorrect guesses will use successivities in $\mathcal{B}$ which turn out to be fake and so will not apply to the correct $\operatorname{oracle} \operatorname{Succ}(\mathcal{B})$; indeed, any $\Gamma$-definitions made "to the right" of the correct guess cannot apply to the oracle $\operatorname{Succ}(\mathcal{B})$.

However, it is possible that we have made a definition of $\Gamma^{\operatorname{Succ}(\mathcal{B})}(i)$ "to the left" of the correct guess, and the use of it may only contain correct information about $\operatorname{Succ}(\mathcal{B})$ and so will apply. But we need to undo this definition (for fixed $i$ ) at most once, namely, when $i$ enters $C$, and at that point, we will act once "to the left of the true path". It is now not hard to see that this action "to the left of the true path" can affect each fixed strategy along the true path at most finitely often, and so we will succeed in building $\mathcal{B}$ and $\Gamma^{\operatorname{Succ}(\mathcal{B})}$ correctly.

Putting these ideas together results in a fairly straightforward infinite-injury priority construction, filling the gap for our missing case.

\section{Acknowledgment}

The authors wish to thank Maxim Zubkov for pointing out the errors corrected in this corrigendum.

\section{References}

[1] J. C. Chubb, A. N. Frolov and V. S. Harizanov, Degree spectra of the successor relation of computable linear orderings, Arch. Math. Logic 48 (2009) 7-13. 
[2] R. G. Downey, S. Lempp and G. Wu, On the complexity of the successivity relation in computable linear orderings, J. Math. Logic 10(1 \& 2) (2010) 83-99.

[3] A. N. Frolov, Presentations of the successor relation of computable linear orderings, Izv. Vyssh. Uchebn. Zaved. Mat. 54(7) (2010) 73-85; translated in Russian Math. (Iz. VUZ) 54(7) (2010) 64-74. 